\title{
Synthesis and Characterization of Nickel-Silver Core-Shell Nanoparticles for Fabrication of Conductive Inks
}

\author{
Anna Pajor-Świerzy ${ }^{1}$, Robert Socha ${ }^{2}$, Radosław Pawłowski ${ }^{3}$, Piotr Warszyński ${ }^{4}$, Krzysztof \\ Szczepanowicz ${ }^{5}$ \\ 1,2,4,5 Jerzy Haber Institute of Catalysis and Surface Chemistry Polish Academy of Sciences, Niezapominajek 8, \\ 30-232 Kraków, Poland \\ ${ }^{3}$ Abraxas Jeremiasz Olgierd, ul. Piaskowa 27, 44-300 Wodzisław Śląski, Poland
}

\begin{abstract}
In recent years, nickel nanoparticles (NPs) as a component of conductive inks have attracted much attention as a low-cost replacement for the currently used silver and gold NPs for fabrication of printed electronic devices. Nickel NPs as a component of conductive inks should be stable against oxidation process at all stages of preparation of conductive patterns: ink formulation and storage, printing, and post-printing treatment. In the present study, the combination of the oxidation resistance of the Ag layer and the conductive properties of the $\mathrm{Ni}$ core was achieved and nickel-silver core-shell (Ni@ Ag) NPs were proposed as the component of a conductive ink. The ink properties were optimized by selecting appropriate wetting agent to obtain high quality ink coating on a glass substrate. To form the percolated network of nanoparticles, which provides transformation of nonconductive films to conductive solid metallic tracks the thermal sintering was performed. We found that the conductivity of ink films was higher after mixing of smaller and bigger core-shell NPs in comparison to ink coating composed only from Ni@ Ag NPs of one size of 70 or $250 \mathrm{~nm}$. After the sintering at $300^{\circ} \mathrm{C}$ for 30 min the conductivity was found to be $17 \%$ of that for the bulk nickel comparing to $11 \%$ of conductivity of film based only on Ni@Ag NPs at the size $250 \mathrm{~nm}$.

The obtained results clearly indicate that prepared Ni@Ag NPs possess promising properties for their application in the printing technology of circuit board. The conductive ink composed of those nanoparticles can be a convenient and low-cost material for the fabrication of printed conductive features.
\end{abstract}

Keywords: air-stable nanoparticles, coating, wetting properties, wet chemical synthesis, thermal sintering 